Ann. Biol. anim. Bioch. Biophys., I975, 15 (3), 503-508.

\title{
ACTIVITÉ SEXUELLE \\ DE MICROCEBUS MURINUS (MILLER, 1777) SOUMIS A DES RÉGIMES PHOTOPÉRIODIQUES EXPÉRIMENTAUX
}

\author{
Arlette PETTER-ROUSSEAUX \\ Laboratoire d'Écologie, \\ Muséum d'Histoire naturelle, \\ 91800 Brunoy
}

RÉSUMÉ

Trois expériences de manipulations photopériodiques sont entreprises pour tester l'influence de la photopériode sur l'activité sexuelle de Microcebus murinus : I) photopériode constante de I 3 h 30 ; 2) rythme de variations trimestrielles ; 3) rythme de variations semestrielles en photopériodes restant inférieures aux durées naturelles $(6 \mathrm{~h}$ à $8 \mathrm{~h}$ ).

Les résultats montrent que la durée d'éclairement est un puissant entraîneur de l'activité sexuelle puisqu'on observe un cycle testiculaire normal en rythme trimestriel; cependant, une activité sexuelle rythmique persiste en photopériode longue, maintenue constante. En photopériodes variant mais restant courtes, l'activité testiculaire tend à être continue. Chez les femelles, on observe peu ou pas d'ovulation en l'absence de séquence suffisante de photopériodes longues. Les photopériodes longues auraient donc pour conséquence d'entraîner d'une part l'ovulation chez les femelles, et d'autre par l'apparition d'un état réfractaire.

L'activité sexuelle de Microcebus murinus est étroitement liée au rythme saisonnier de variations photopériodiques, les jours croissants entraînant l'activité (accompagnée d'une chute de poids corporel) et les jours décroissants entraînant le repos et l'engraissement. Un rythme de variations photopériodiques de 6 mois au lieu d'un an entraîne deux saisons d'activité sexuelle par an (PETTER, 1972). Tout ceci indépendamment de l'amplitude des variations et de la longueur des photopériodes, puisqu'on observe le même phénomène en jours croissants entre 8 heures et Io heures (janvier-février à Paris) ou entre II heures et I2 heures (juillet-août à Madagascar).

Nous avons cherché à approfondir notre connaissance sur l'importance de l'entraînement photopériodique dans l'activité sexuelle en soumettant des animaux mâles et femelles à trois types de manipulations photopériodiques : 
I. Éclairement constant de 13 h 30 par jour ;

2. Variation, de période de 3 mois et d'amplitude de 4 heures;

3. Variation semestrielle, mais en photopériode inférieure aux durées naturelles.

\section{MATÉRIEL, ETT MÉTHODES}

Les Microcèbes sont installés seuls ou par deux dans des cages de grillage de 50 cm de côté garnies de nichoirs en bois. Ils sont nourris de fruits, lait miel, vers de farine, viande crue hachée. La température de 1'animalerie est constamment voisine de $25^{\circ} \mathrm{C}$. Les animaux ont été pesés chaque mois au moins; on a, d'autre part, mesuré la longueur des testicules, à 2 mm près, et noté les aestrus des femelles (gonflement de la peau sexuelle et ouverture vaginale).

I. Nous avons soumis à une durée journalière de $\mathrm{I} 3 \mathrm{~h}$ 30 de lumière des Microcèbes ayant subi au préalable, pour les uns un rythme annuel de variations photopériodiques, pour les autres un rythme semestriel. L'expérience a duré $\mathbf{4} 4$ mois pour les premiers $(2$ ot et 4 o) et I 6 mois pour les seconds $(3$ ot 9 o).

2. Nous avons choisi un rythme de trois mois, pendant lequel la photopériode était fixée pour une semaine à $I_{4}$ heures de lumière. Elle décroissait ensuite par paliers successifs, à raison de 20 minutes chaque semaine, pour atteindre $I_{0} \mathrm{~h} 20$ avant de remonter brutalement à I4 heures, 3 mois plus tard. 2 o et 7 우 ont été soumis à cette expérience qui a duré 14 mois, au bout desquels la photopériode est restée fixée à I 4 heures.

3. L'expérience a porté sur 7 ô et 8 ㅇ, soumis pendant I 4 mois à un système de variations photopériodiques dans lequel les photopériodes variaient régulièrement mais entre $6 \mathrm{~h}$ et $8 \mathrm{~h}$ seulement.

\section{RÉSULTATS}

Les résultats correspondant aux trois expériences sont groupés dans les trois graphiques.

I. On constate que sous éclairement constant de 13 h 30 , par jour, 1'activité testiculaire demeure rythmique : à des périodes d'activité pendant lesquelles le testicule atteint sa taille maximum succèdent des périodes de repos. Les animaux ne sont pas synchronisés au moment de la reprise, le décalage pouvant atteindre 3 mois ; tous cependant sont en activité d'octobre à décembre. Il existe toujours une correspondance étroite entre la variation de poids et le développement testiculaire (fig. I).

Les femelles ayant subi un rythme semestriel présentent des variations de poids beaucoup moins nettes et les cestrus s'étalent sur plusieurs mois après que l'ensemble des autres animaux soit entré en repos. La mortalité par ailleurs, a été très élevée dans ce groupe (5 9 sur 9 au cours des 16 mois).

2. Quand la période est réduite à 3 mois, en maintenant une photopériode longue de $\mathrm{I} 4 \mathrm{~h}$ pendant une semaine et en l'amenant progressivement à Io $\mathrm{h} 20$ en $\mathrm{I} 2$ semaines, le testicule subit des cycles d'activité parfaitement synchronisés par le retour à I4 h de lumière. Pour la femelle, la réponse est différente en ce sens que la durée d'une semaine d'éclairement de I4 h est insuffisamment longue pour entraîner l'ovulation ; ce n'est qu'en prolongeant cette durée jusqu'à trois semaines que l'on obtient l'ovulation (fig. 2). 

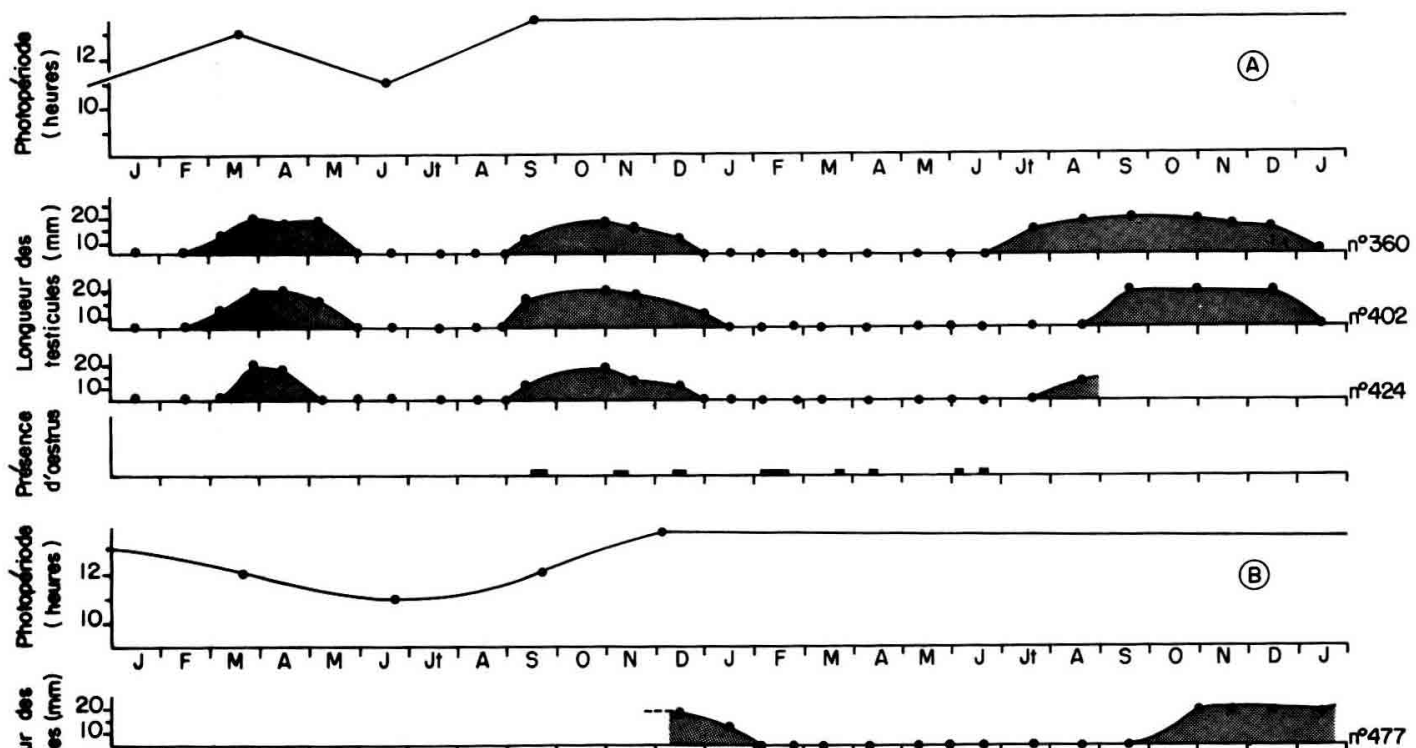

(A) -Animaux préalablement en rythme semestriel

(B) - Animoux préalablement en rythme annuel

FIG. 1. - Longueur des testicules en mm, et présence äcostrus chez des Microcèbes soumis à une photopériode constante de 13 h 30 après un rythme semestriel de variations photopériodiques (A); après un rythme annuel (B)
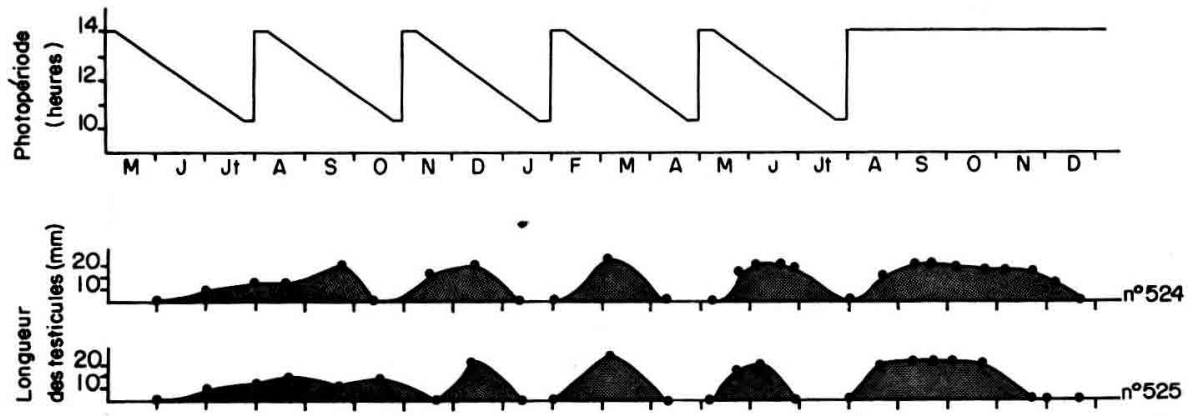

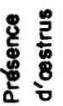

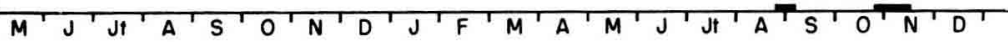

FIG. 2. - Longueur des testicules en inm, et présence d'astrus chez des Microcèbes soumis à un rythme trimestriel de variations photopériodiques,

suivi d'une photopériode constante de $14 \mathrm{~h}$ 
3. Sous un éclairement de faible durée journalière mais présentant des variations d'amplitude régulières, le testicule peut ne pas régresser ; 5 ô sur 7 présentent des variations, mais non synchronisée, ni entre eux ni avec les variations photopériodiques; leurs périodes de repos ne durent pas plus d'un mois dans la plupart des cas ; enfin, chez 2 individus, l'activité qui a débuté au bout de deux mois persiste jusqu'à la fin de l'expérience (I2 mois de développement testiculaire) (fig. 3).
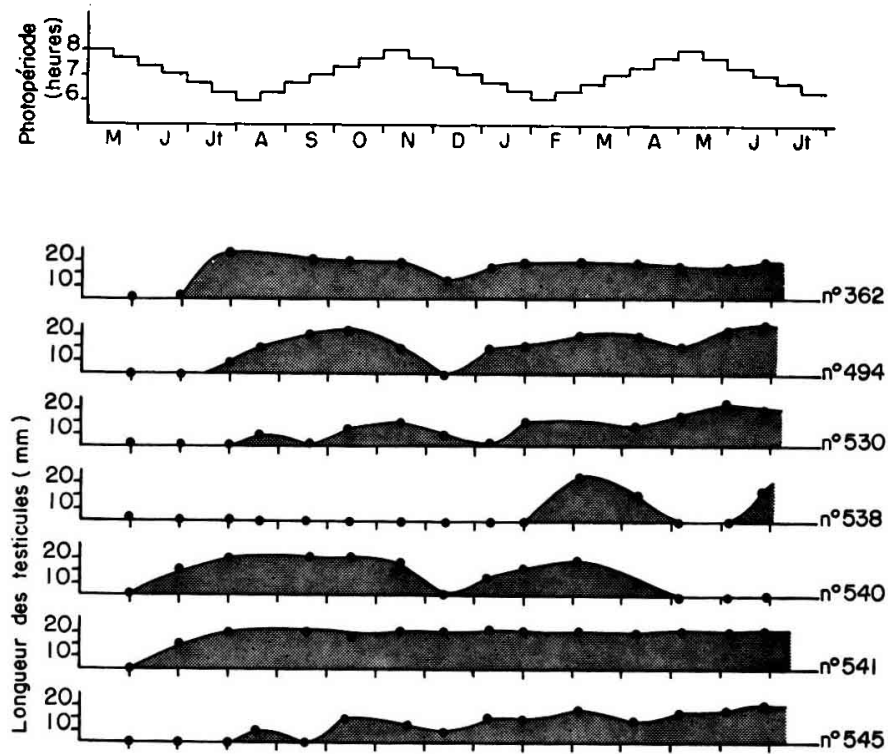

FIG. 3. - Longueur des testicules en mm, chez des Microcèbes soumis à un rythme semestriel de variations photopériodiques dans lequel la photopériode varie entre $6 h$ et 8 h seulement

Ce comportement contraste avec celui des femelles : sur 8 individus, une seulement entre en ostrus.

\section{DISCUSSION}

Le petit nombre d'animaux soumis aux expériences incite à conclure avec prudence; quelques points, cependant, paraissent bien établis.

La première expérience montre clairement que bien qu'une photopériode croissante ait induit 1'activité testiculaire, celle-ci ne se maintient pas si la photopériode longue est maintenue constante. Les phases de repos et d'activité qui se succèdent alors n'ont pas de relation apparente avec des variations saisonnières du milieu, les facteurs d'hygrométrie, température, alimentation étant relativement constants.

Chez les mâles ayant subi préalablement un rythme semestriel de variations photopériodiques, il y a tendance à l'allongement des phases d'activité et de repos par rapport au rythme imposé précédemment.

I a deuxième expérience montre que la durée d'éclairement est un puissant entraîneur de l'activité sexuelle du Microcèbe, puisqu'un cycle testiculaire normal 
et régulier peut être observé dans un rythme de 3 mois. L'arrêt temporaire de l'activité sexuelle sous éclairement constant conduit donc à l'idée, soit qu'il existe un rythme endogène de la fonction gonadotrope, soit qu'il apparait après une période d'acti vité, une période de repos obligatoire, qui correspondrait à une période réfractaire.

Nous avions remarqué qu'en rythme semestriel, le délai entre le début de 1'accroissement des jours et l'apparition du développement testiculaire était plus long qu'en rythme annuel : un mois et demi au lieu d'une quinzaine de jours (PETTER, I972). Dans l'expérience présente, ce délai est de nouveaut très court : à peine ${ }_{5}$ jours. On peut penser alors que ce délai est dû à un état réfractaire qui aurait ou non le temps de s'instaurer et de prendre fin selon les régimes utilisés : en rythme annuel, il prendrait naissance en jours décroissants et disparaitrait en jours courts avant la reprise de la stimulation; en rythme semestriel, il n'aurait pas le temps de disparaitre avant la reprise des jours croissants; dans le rythme trimestriel que nous avons utilisé, il n'aurait pas le temps de s'instaurer, la séquence de jours longs de une semaine étant insuffisante. Il semblerait donc qu'un état réfractaire ne puisse se développer qu'en jours décroissants succédant à des jours longs suffisamment nombreux.

Chez les femelles, il semble bien que l'œestrus ne puisse avoir lieu qu'après un minimum de jours longs et ne décroissant pas, puisque 3 semaines suffisent quand la photopériode est stabilisée à $\mathrm{I} 4$ heures, alors qu'un mois et demi de jours d'une durée d'au moins $\mathrm{r} 2$ h 30 mais décroissants n'entraîne rien. Rappelons qu'en rythme semestriel, l'œstrus est atteint plus tôt qu'en rythme annuel et que ceci peut être dû au fait que l'accroissement des photopériodes étant plus rapide, les jours longs nécessaires sont atteints plus vite. Seule la connaissance du niveau de sécrétion des stéroïdes ovariens pourrait permettre une interprétation de ce comportement particulier de la femelle.

La troisième expérience apporte des éléments supplémentaires aux deux questions de l'établissement d'une période réfractaire et de la nécessité des jours longs dans le cas des femelles.

Rappelons d'abord que la variation photopériodique utilisée semble ne pas influencer le développement testiculaire, puisqu'elle n'entraîne pas de synchronisation entre les différents individus et qu'il n'y a pas de correspondance entre jours croissants et accroissement testiculaire et inversement, alors que c'était le cas quand les animaux étaient soumis à un régime de variations de même amplitude et de même période, mais dont les variations se situaient autour de I 2 heures.

En photopériodes rythmiques mais courtes, on observe une réduction des périodes de repos : si l'on considère la pérıde de I 2 mois comprise entre le I er juillet I 972 et le $\mathrm{I}^{\mathrm{e}}$ juillet $\mathrm{r} 973$, on peut compter une moyenne de 8,85 mois d'activité testiculaire par animal ; des mesures faites en rythme annuel (sur le même nombre de 7 o) donnent les moyennes suivantes : 6,85 et 7,43 , l'activité du même animal ne dépassant jamais 9 mois. On peut penser, ici encore, que l'absence de jours longs aboutit à une réduction de l'état réfractaire. Chez les femelles, au contraire, on ne constate, dans cette troisième expérience, qu'un seul cas d'œstrus; on retrouve donc la nécessité constatée dans la deuxième expérience, d'une séquence minimum de jours longs pour qu'apparaisse 1'œstrus.

I1 parait bien établi maintenant que la stimulation qui entraîne 1'activité sexuelle n'est pas due à la photopériode elle-même, mais à l'action de la lumière à un moment donné, correspondant à une phase de sensibilité de l'organisme. Grocock 
et Clarke (I974), par exemple, estiment que, chez le campagnol, le développement testiculaire serait stimulé par l'excitation d'une période sensible située I 2 heures après l'allumage. Dans notre expérience, le moment propice à la stimulation du développement testiculaire serait donc fréquemment exposé, même en jours très courts ; on peut penser que le moment propice à l'établissement d'un état réfractaire ne le serait au contraire, qu'en jours longs ; de même, le moment propice à la préparation de l'ovulation ne serait pas stimulé dans les expériences rapportée ici. Ceci met en évidence la dissociation entre les conditions requises pour le départ de l'activité sexuelle et celles qui sont indispensables à l'aboutissement qu'est l'ovulation chez la femelle. Il est probable en effet, que le développenent testiculaire, comme la maturation de l'ovocyte, ne dépendant pas de la stimulation d'une seule période, photosensible.

Reçu pour publication en février 1975.

\section{SUMMARY}

SEXUAL, ACTIVITY OF MICROCEBUS MURINUS (MILLER, I777) UNDER EXPERIMENTAL PHOTOPERIODS

The sexual activity of Microcebus murinus is strongly correlated with seasonal rhythm of daylight variation. In this study, it is observed under 3 experimental photoperiods :

I) 2 males and 4 females, previously kept on annual rhythm, are subjected to a constant photoperiod of $\mathrm{I}_{3} \mathrm{~h} 30 \mathrm{~L}$ - Io h $30 \mathrm{D}$ for $\mathrm{I} 4$ months ; 3 males and 9 females, previously kept on a 6-month rhythm, are subjected to the same photoperiod for 16 months ;

2) 2 males and 7 females are given a 3 -month rhythm of photoperiodic variations for 14 mnoths; the photoperiod, fixed at I4 hours for I week, de creases by successive stages during the following i I weeks, to reach io $h 20$. It then rises suddenly to I 4 hours. At the end of I 4 months, the photoperiod stays definitively fixed at 14 hours ;

3) 7 males and 8 females are subjected to a 6-month rhythm of photoperiodic variations in which the daylengths are shorter than natural ones, varying between $6 \mathrm{~h}$ and $8 \mathrm{~h}$ only.

The results show that I) sexual activity stays rhythmic in constant photoperiod. Although induced by increasing days, it does not persist under long, invariable daylengths ; z) daylength is a strong " timer " of sexual activity because a normal, regular testicular cycle is observed under the 3 -month rhythm. Arrest of sexual activity under an invariable daylength suggests that there is endogenous rhythm of gonadotropin function, or a period of compulsory rest corresponding to a refractory period, which appears after a period of activity.

No estrus is observed in females during the second experiment, but all enter into estrus 3 weeks after stabilization of the daylength at $I_{4} \mathrm{~h}$. It would seem that a minimal duration of long, invariable daylength is necessary to induce ovulation. estrus.

This is corroborated by the third experiment in which only one female out of eight presents

3) Under a short, regularly changing daylength, the testicular regression phases tend to decrease, and even disappe ar in some cases. It is concluded that long daylengths favorize appearance of a refractory period. This conclusion is supported by the results of the second experiment, in which long, invariable daylengths only last one week out of 12 and 14 -hour photoperiodic stimulation induces a very prompt response.

\section{RÉFÉRENCES BIBLIOGRAPHIQUES}

Grocock C. A., Clarke J. R., I974. Photoperiodic control of testis activity in the vole, Microtus agrestis. J. Reprod. Fert., 39, 337-347.

Petter-Rousseaux A., I972. Application d'un système semestriel de variations de la photopériode chez Microcebus murinus (Miller, i777). Ann. Biol. anim. Bioch. Biophys., 12, 367-375. 